\title{
Dental Hygienists' Awareness and Support for Existing and New Oral Health Workforce Models
}

\author{
Jennifer D. Sanders ${ }^{1} \cdot$ Sena Narendran ${ }^{1}$
}

Published online: 30 July 2015

(C) Springer International Publishing AG 2015

\begin{abstract}
The study investigated dental hygienists' scope of services and level of satisfaction as well as their awareness and support to the existing and proposed oral health workforce models: expanded function dental auxiliary (EFDA), dental therapist (DT), and advanced dental hygiene practitioner (ADHP). The study sample consisted of all 676 dental hygienists from Cuyahoga County, Ohio. Data were collected by a self-administered mail questionnaire that assessed hygienists' scope of services, levels of satisfaction with the services, and their awareness as well as support for workforce models; the last two were assessed on a scale of $1-10$. The effective response rate to the survey was $26 \%$. Respondents' average ratings for the knowledge levels for DT, ADHP, and EFDA were as follows: $2.8 \pm 2.6,4.0 \pm 3.4$, and $8.1 \pm 2.6$. Hygienists were most supportive of the EFDAs (7.7 \pm 2.9$)$, followed by ADHP (7.0 \pm 3.4$)$, and DT (4.0 \pm 3.4$)$. Findings indicate a need to improve respondents' awareness of the dental therapist position.
\end{abstract}

Keywords Oral health workforce - Dental hygienist . Expanded function dental auxiliary · Dental therapist, advanced dental hygiene practitioner $\cdot$ Community dental health coordinator

This article is part of the Topical Collection on Dental Public Health

Sena Narendran

sxn100@case.edu

1 Department of Community Dentistry, School of Dental Medicine, Case Western Reserve University, 2124 Cornell Road, Cleveland, OH 44106-4905, USA

\section{Introduction}

The Surgeon General's Report brought into light and into the minds of the oral health community the disparities in the availability and accessibility of oral health services, depending on ethnicity, age, socioeconomic status, and location [1]. It is believed that currently a larger number of dentists are retiring than new dentists will be graduating, which has led certain organizations to realize that there is a shortage of dentists in many areas and that solutions must be found to improve the situation. In order to alleviate these workforce shortages, new oral health provider positions have been proposed and/or introduced in the USA; the new positions include dental health aide therapist (DHAT), community dental health coordinator (CDHC), and advanced dental hygiene practitioner, (ADHP) [2].

The American Dental Hygienist's Association has developed the ADHP position, which is a 2-year master's level program. The program will permit the ADHP to perform some procedures unsupervised and some others under the general supervision of a dentist; the ADHP will be able to do restorations and simple extractions [3•]. The advanced dental hygienist practitioner's scope of practice includes that of dental therapists and a few additional functions, as well as that of registered dental hygienists. Advanced dental therapist (ADT), a new position based on the ADHP model with slight variations through legislative debate and compromise, is currently in place in Minnesota. ADT training was first implemented in September 2009 [2].

The dental therapist position that is in place in Alaska was adopted from the New Zealand dental nurse model, which recognizes dental nurses as members of the dental care team who treat children exclusively [4]. Dental nurses have been practicing in New Zealand, Australia, Malaysia, and a few other countries since the 1950 s, providing services to children 
in underserved areas and schools. Dental nurses or dental therapists are also currently working in Australia, Britain, and Canada. The Canadian model of dental therapist varies slightly from the New Zealand dental nurse model in that Canadian dental therapists provide care not only to children but to adults as well. More recently, however, certain countries have expanded dental nurses' functions to treat adults as well. In both the Canadian and New Zealand models, all procedures must be performed under the supervision of a dentist [5]. There are many proponents of bringing dental therapists to the continental USA, in order to increase the availability of oral health care for children and to improve access to dental care for the underserved $[2,5,6,7]$.

The proposed DT models vary in the scope of functions and levels of required supervision based on practice location, and these positions have been referred to by different names. In Alaska, DTs are known as dental health aide therapists (DHAT), who, with 2 years of training, perform many of the same procedures as a registered dental hygienist. DHATs also perform additional procedures such as diagnosing and restoring dental caries and extracting teeth. Alaska graduated its first class of DHATs in December 2008, and the primary focus of these DHATs is to address the dental needs of native populations [5].

The American Dental Association is a strong proponent of another new model, the community dental health coordinator (CDHC), whose training is 18 months long [2]. Three dental schools have implemented the CDHC training, which are as follows: Temple University and Universities of Oklahoma as well as California at Los Angeles. The programs are open to high school graduates, and the goal is again to improve access to dental care by eliminating certain barriers including, but not limited to, culture, awareness, and socioeconomic status. CDHCs' functions include dental health education, advocacy, and preventive services, and they mainly triage patients to dentists. In addition to clinics, CDHCs will work in schools as well as places of worship.

Another position is the Expanded Function Dental Auxiliaries (EFDA), who have been practicing in many states, including Ohio, since 1980. Services provided by EFDAs vary in scope from state to state. In Ohio, EFDAs work under direct supervision of dentists. In addition to the usual scope of a dental assistant's duties, EFDAs can place pit-and-fissure sealants and do simple restorations. The EFDA training is usually $6-9$ months in length, depending on the school administering the program [8].

Anderson and Smith investigated dental hygienists' opinions about the Bachelor of Science degree in Dental Hygiene and the Oral Health Practitioner (OHP) position in Minnesota. Through legislative amendments since the publication of the study, OHP is now referred to as dental therapist or advanced dental therapist [9]. The authors found that the majority of the dental hygienists thought that the OHP position would improve access to care as well as advance the profession of dental hygiene. Further, the respondents acknowledged that the primary barriers to becoming an OHP were time and finances [9]. Most respondents were in support of the Bachelor of Science in Dental Hygiene, particularly the younger respondents; the perception was that additional training would "improve professional competence and professional recognition." Okwuje and group found that a majority of academic dental hygiene program directors felt that it was important to support and promote the ADHP model [10].

A limited number of studies have investigated various stakeholders' knowledge and opinions of the new and existing oral health workforce models. These include the following: deans of dental schools [11•], dental school faculty members [12•], and dental students [13]. Overall, the literature indicates that dental hygienists are in support of further education of the ADHP or ADT model, as it is now known in Minnesota. Because new workforce models have been proposed or created recently, there is very limited literature regarding dental hygienists opinions and perceptions about the new positions. The purpose of the study was to investigate dental hygienists' satisfaction and comfort levels in performing procedures in their scope of clinical practice and which of the other dental professionals could perform the same. Furthermore, the study assessed dental hygienists' awareness and opinions of some of the existing EFDA positions and newly proposed dental care workers such as the dental therapists and ADHP.

\section{Methods}

The Institutional Review Board at the university with which the authors are affiliated approved the study. The study sample consisted of all 676 licensed dental hygienists in Cuyahoga County, Ohio. A list of licensed dental hygienists in Cuyahoga County was obtained from the Ohio State Board of Dentistry. The questionnaire, along with a cover letter and a postagepaid return envelope, was mailed to all the hygienists in the county. After 4 weeks of the initial mailing, a second questionnaire was sent to the non-respondents of the first mailing.

Data were collected by self-administered mail questionnaires that consisted of 25 items of which 23 were precoded and two were open-ended. The questionnaire assessed the scope of services provided by the dental hygienists, their levels of satisfaction in providing such services as well as whom they think should be providing such services. The survey instrument also investigated respondents' awareness and support for the dental therapist (DT), EFDA, and ADHP positions. Respondents denoted their levels of satisfaction, awareness, and support on a scale of 1 to 10 with 10 being the highest ranking.

When the questionnaires were returned, data were appropriately coded and entered into the Statistical Packages for 
Social Science $\left(\right.$ SPSS $\left.^{\circledR}\right)$ editor. A majority of the questionnaire data were categorical and are presented mainly in the form of percentages/proportions and/or frequency distribution. Medians, means, and standard deviations were computed for continuous variables such as: years in practice, number of specific procedures per month, levels of knowledge, and support. Analytical statistics included computation of odds ratio and a chi-squared statistic, which assessed the relationship between independent (years in practice, awareness of positions, etc.) and dependent variables such as levels of satisfaction and comfort in performing the procedures as well as level of support for the proposed and existing positions.

For the purpose of analytical tests, knowledge and support level variables were recoded into two categories based on the mean score for each variable rounded to the nearest whole number. The cutoff points for the knowledge levels were as follows: dental therapist -3.0, ADHP -4.0, and EFDA -8.0. Respondents above the cutoff point were classified as having a "good" knowledge level and those below, "poor." Similarly, the respective demarcation score for the levels of support for dental therapist, ADHP, and EFDA were 4.0, 7.0, and EFDA, 8.0. Those above these scores were deemed to be more supportive of the respective position, and those below were less supportive. For the item on the likelihood of pursuing continuing education to become an ADHP, the cutoff score was 5.0; those with scores above five were more likely to pursue further education, and those below five were less likely. We utilized the dichotomous classification among the variables for chi-squared test and to compute odds ratios and $95 \%$ confidence intervals to assess the magnitude of the associations between respective independent and dependent variables. The level of significance for the analytical tests, alpha, was set at less than or equal to 0.05 .

\section{Results}

After two mailings, a total of 158 useable questionnaires were returned and 61 were returned as non-deliverable for different reasons, for an effective response rate of $26.1 \%$. Respondents have been practicing anywhere from 1 to 47 years with a mean of $27.2 \pm 11.7$ years.

Prophylaxis and dental health education were the two most commonly performed services by the study subjects: 120 and 110 times/month. While subjects were most satisfied in performing the above services, they were least satisfied with "repair, construction, and finishing of metallic and plastic prosthetic devices" and "intra-oral bite registrations for diagnostic models." The lowest levels of satisfaction were denoted by scores of $3.9 \pm 4.4$ and $5.2 \pm 4.4$, respectively, on a scale of 1 to 10 with 10 being the highest level of satisfaction. The procedures that the hygienists were least satisfied with were also the least performed along with "application and removal of periodontal dressings" with the scores of $1.3 \pm 4.3$ and $0.9 \pm 4.0$.

In general, less than $50 \%$ of the respondents felt that dental therapists will be able to perform the functions within the scope of dental hygiene practice. At the highest level, nearly $44 \%$ of respondents felt that dental therapists should be able to apply fluoride agents to teeth as well as adopt infection control procedures. At the lower end of the spectrum, $14.5 \%$ of respondents felt that dental therapists can "repair, construct, and finish metallic, and plastic prosthetic devices" and $21.5 \%$ felt the same about "intra-oral bite registrations for diagnostic models." Only $22 \%$ of respondents believed that dental therapists could administer block anesthesia within the oral cavity.

Figure 1 illustrates dental hygienists' level of support for the proposed and existing oral health workforce models; respondents were mostly in support of the EFDA position (mean $7.7 \pm 2.9$ ), followed by ADHP (mean 7.0 \pm 3.4 ) and finally, DT (mean 4.0 \pm 3.4 ). The level of support for the DT was significantly lower than that for either EFDA or ADHP. Respondents were also most informed about the EFDA position $(8.1 \pm 2.6)$, followed by ADHP (4.0 \pm 3.4$)$ and finally, DT (2.8 \pm 2.6$)$.

The level of support for existing and new workforce models increased with respondents' knowledge/informed level of respondents (Table 1). Respondents with good knowledge of DT were nearly three times as likely to support DT (odds ratio $(\mathrm{OR})=2.9,95 \%$ confidence interval $(\mathrm{CI})=1.5$, 5.9). Similarly, the respondents with good knowledge of dental therapists' were four and six times more likely to support ADHP $(\mathrm{OR}=4.0,95 \% \mathrm{CI}=2.0,8.2)$ and $\mathrm{EFDA}(\mathrm{OR}=5.6$, $95 \% \mathrm{CI}=2.8,11.2)$.

Respondents' levels of interest in further advancing their careers to become advanced dental hygiene therapists were strongly associated with their knowledge levels as well as support for the various oral health workforce models as shown in Table 2. Dental hygienists who were more inclined to pursue higher education to become ADHP were also more informed of dental therapists, ADHP and EFDA (Table 2). Those who were more likely to seek additional education were three times more knowledgeable of dental therapists compared to the hygienists who were less inclined to do so $(\mathrm{OR}=2.8,95 \% \mathrm{CI}=1.4,5.6)$. Dental hygienists with higher levels of interest in becoming ADHPs were also more knowledgeable about ADHPs $(\mathrm{OR}=2.6,95 \% \mathrm{CI}=1.3,5.1)$. The level of interest in pursuing ADHP education, however, was not associated with the respondents' knowledge levels of EFDAs $(\mathrm{OR}=2.1,95 \% \mathrm{CI}=0.8,3.2)$.

Table 2 depicts the association between respondents' desire to pursue the ADHP education and support for the new model of oral health workforce, DT. Dental hygienists who were more likely to pursue such education were twice as likely as their less inclined counterparts to support dental therapists $(\mathrm{OR}=2.2$, $95 \% \mathrm{CI}=1.1,4.3$ ); their level of support for ADHP was nearly 
Fig. 1 Respondents informed level and support for existing and new workforce models

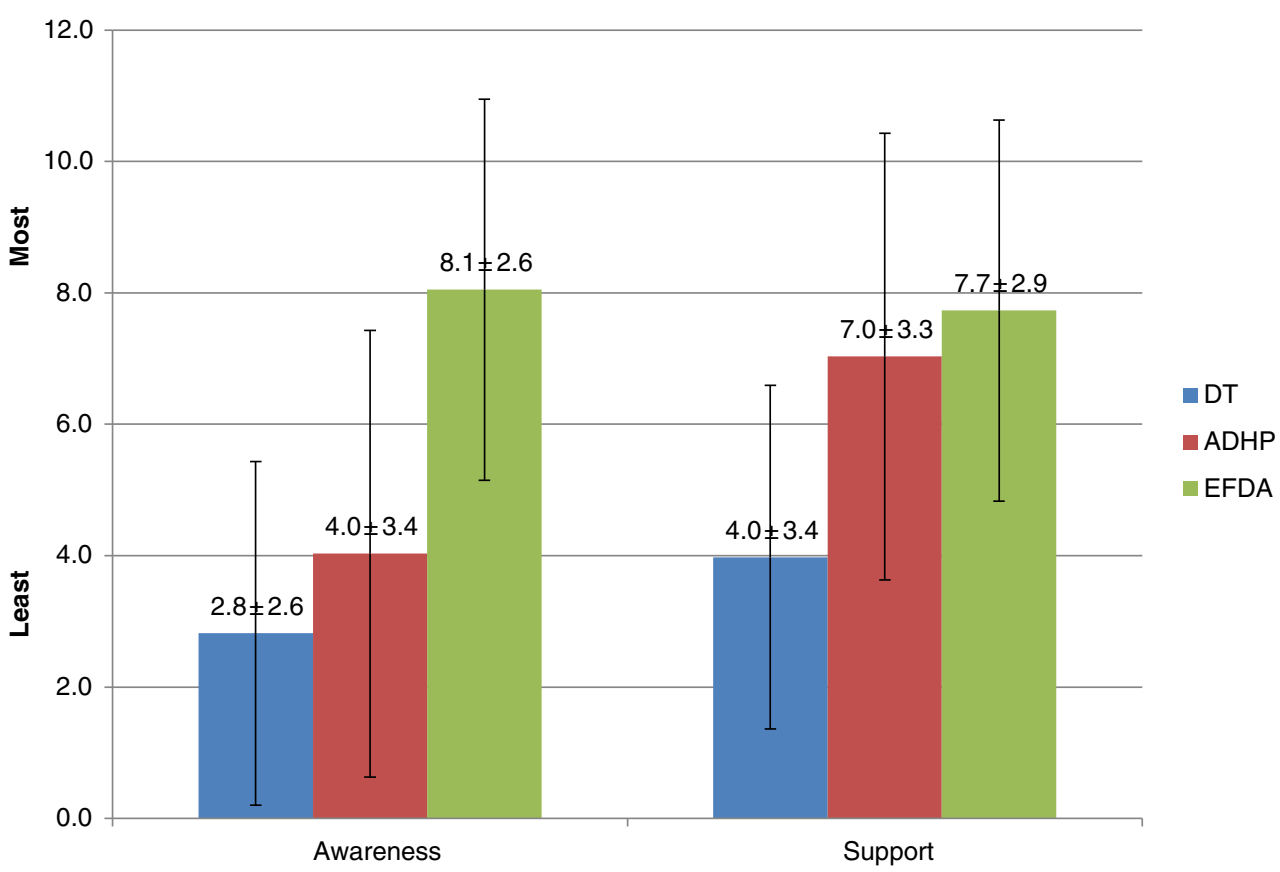

13 times higher than those with less academic aspirations $(\mathrm{OR}=12.5,95 \% \mathrm{CI}=5.1,30.1)$. The level of support for EFDAs by respondents aspiring to become ADHPs, however, was not statistically significant $(\mathrm{OR}=1.5,95 \% \mathrm{CI}=0.8,2.8)$.

\section{Discussion}

This is one of the first studies to investigate dental hygienists' awareness and support for some of the newly proposed dental provider positions and existing dental personnel such as the Expanded Function Dental Auxiliary. Thus, one of the strengths of this project is that it has allowed us to get some insight into the beliefs of dental hygienists regarding other dental personnel, which was previously unknown. The study sample, despite being limited to only one county in the state of Ohio, included all the registered dental hygienists in the County, thus eliminating any sampling errors; the total population of hygienists in the

Table 1 Association between respondents' knowledge levels and their support for the existing and new oral health workforce models

\begin{tabular}{llllll}
\hline Knowledge & & \multicolumn{3}{l}{ Support } & \\
\cline { 3 - 6 } & & More & Less & OR & $95 \%$ CI \\
\hline \multirow{2}{*}{ DT } & Good & 28 & 22 & 2.9 & $1.5,5.9$ \\
& Poor & 30 & 69 & & \\
ADHP & Good & 48 & 15 & 4.0 & $2.0,8.2$ \\
& Poor & 39 & 49 & & \\
EFDA & Good & 63 & 28 & 5.6 & $2.8,11.2$ \\
& Poor & 19 & 47 & & \\
\hline
\end{tabular}

OR: odds ratio, $95 \%$ CI: confidence interval for the odds ratios, $95 \%$
Table 2 Association between respondents' interest in career development (CA) and their knowledge of and levels of support for the existing and new workforce models

\begin{tabular}{|c|c|c|c|c|}
\hline Interest in CA & Knowledge & & OR & $95 \% \mathrm{CI}$ \\
\hline & DT & & & \\
\hline & Good & Poor & & \\
\hline More likely & 28 & 33 & 2.8 & $1.4,5.6$ \\
\hline \multirow[t]{3}{*}{ Less likely } & 21 & 68 & & \\
\hline & ADHP & & & \\
\hline & Good & Poor & & \\
\hline More likely & 34 & 29 & 2.6 & $1.3,5.1$ \\
\hline \multirow[t]{3}{*}{ Less likely } & 28 & 62 & & \\
\hline & EFDA & & & \\
\hline & Good & Poor & & \\
\hline More likely & 41 & 22 & 2.1 & $0.8,3.2$ \\
\hline \multirow[t]{3}{*}{ Less likely } & 48 & 42 & & \\
\hline & Support for DT & & & \\
\hline & Good & Poor & & \\
\hline More likely & 32 & 30 & 2.2 & $1.1,4.3$ \\
\hline \multirow[t]{3}{*}{ Less likely } & 29 & 60 & & \\
\hline & Support for ADHP & & & \\
\hline & Good & Poor & & \\
\hline More likely & 53 & 7 & 12.5 & $5.1,30.1$ \\
\hline \multirow[t]{3}{*}{ Less likely } & 34 & 56 & & \\
\hline & Support for EFDA & & & \\
\hline & Good & Poor & & \\
\hline More likely & 36 & 27 & 1.5 & $0.8,2.8$ \\
\hline Less likely & 44 & 48 & & \\
\hline
\end{tabular}

OR: odds ratio, $95 \%$ CI: confidence interval for the odds ratios, $95 \%$ 
county rather than a non-probability sample. Despite the minimal or no sampling errors, findings of the study must be interpreted with caution because of the relatively low response rate.

Respondents in the study were largely uninformed about the ADHP and DT positions as indicated by relatively low mean scores compared to EFDAs. The EFDA position has been in place for more than 25 years compared to ADHP and DT, and each of which has been in place for less than 5 years. Naturally, respondents were more familiar with the position that has been in place for a longer period of time than the new ones. Thus, we believe that this lack of familiarity had resulted in lower levels of support for ADHP and DT. A few respondents commented that this survey was the first time they had ever heard of the ADHP and DT positions. Many respondents commented that either they worked as EFDAs before becoming dental hygienists or they had worked with EFDAs, which may be another reason for the higher information level and support for the EFDA position. Though there is still a reasonable amount of support for the ADHP position, the dental therapist position did not capture much support from the dental hygienists in Cuyahoga County. These findings indicate a need to increase the awareness of the new oral health workforce models such as DT and ADHP to dental hygienists, and we believe this responsibility belongs to the national, state and local dental hygiene and public health associations.

The association between the awareness of the proposed and existing dental personnel positions and the support for the positions were confirmed by statistically significant odds ratios. The higher the awareness of the position, the greater the magnitude of the association; the odds ratios were the highest for EFDA, followed by ADHP and the lowest for dental therapist. The associations between the awareness of the positions and levels of support further reiterate the need to increase the knowledge level of dental hygienists about the proposed new positions. The awareness and support of the dental hygienists for these positions are vital for successful implementation, which will then help us attain the common goal of improving access to dental care, particularly to the underserved populations $[2,5,6]$.

It was heartening to note that a substantial proportion of the respondents expressed interest in pursuing further education to become advanced dental hygiene practitioners (ADHP), which would be beneficial to the profession of dentistry and public at large. Since the goal of the proposed new workforce models such as the ADHP and DT is to improve access to dental care, particularly to the underserved, an increase in the number of dental providers will likely improve the access to care $[5,6]$. The present study also found a positive association between the likelihood of pursuing additional education to become ADHP and the level of support for the new positions such as the ADHP and DT. The increased support for the new positions augurs well for certain segments of the dental profession to support each other and work towards the common goal of improving access to dental care.
In addition to the relatively low response rate, there were other inherent limitations in the present study. It was limited to only one county in a state, thus compromising the external validity of the findings. Future studies with a nationally representative sample of dental hygienists will circumvent the compromised external validity. This study was also limited in that we only included items about only three positions in the questionnaire and did not include the community dental health coordinator (CDHC) position developed by the American Dental Association. In addition, extending research not only nationally but also to nations where they have had dental therapists for many years could shed new light on issues related to one of the new workforce models in the USA. It would also be helpful to know how the current EFDAs feel about the new workforce models.

\section{Conclusions}

Findings from the present study indicate a need to improve dental hygienists' knowledge/awareness of the newly proposed oral health workforce models: DT and ADHP. Such an improvement would likely increase hygienists' support for the new positions and perhaps increase the number of dental hygienists willing to advance their careers to become ADHPs. Mutual support and respect among various dental care providers is essential to attain the common goal of improving access to dental care, which would then lead to better oral health of the public.

\section{Compliance with Ethics Guidelines}

Conflict of Interest Jennifer D. Sanders and Sena Narendran declare that they have no conflict of interest.

Human and Animal Rights and Informed Consent The Institutional Review Board at Case Western Reserve University approved this study.

\section{References}

Papers of particular interest, published recently, have been highlighted as:

- Of importance

1. U.S. Department of Health and Human Services. Oral Health in America: A Report of the Surgeon General. Rockville, Md., U.S. Department of Health and Human Services, National Institute of Dental and Craniofacial Research, National Institutes of Health, 2000. NIH Publication 00-4713. 
2. McKinnon M, Luke G, Bresch J, Moss M, Valachovic RW. Emerging allied dental workforce models: considerations for academic dental institutions. J Dent Educ. 2007;71(11):1476-91.

3. American Dental Hygienists Association. (2009). The history of Introducing a New provider in Minnesota. Retrieved November 15, 2014. http://www.adha.org/downloads/MN_Mid-Level_ History_and_Timeline.pdf. This document succinctly outlines the development of ADHP and OHP in Minnesota; the latter subsequently evolved into dental therapist (DT) and advanced dental therapist (ADT). The evolution of these positions between 2006 and 2014 is illustrated with a clear time-line.

4. Nash DA. Adding dental therapists to the health care team to improve access to oral health care for children. Academic Pediatrics. 2009;9(6):446-51.

5. Quiñonez CR, Locker D. On the pediatric oral health therapist: lessons from Canada. J Public Health Dent. 2008;68(1):53-6.

6. National aboriginal health organization, the profession of dental therapy; discussion paper, 2003. http://www.naho.ca/english/pdf/ research_dental.pdf.

7. Nash DA, Nagel RJ. Confronting oral health disparities among American Indian/Alaska native children: the pediatric oral health therapist. Am J Public Health. 2005;95(8):1325-9.

8. Commission on Dental Testing in Ohio. (2008). How to become an EFDA? Retrieved November 18, 2014. http://www.codtohio.org/ information/how-to-become-an-efda
9. Anderson KL, Smith BS. Practicing dental hygienists' perceptions about the bachelor of science in dental hygiene and the oral health practitioner. J Dent Educ. 2009;73:1222-32.

10. Okwuje I, Anderson E, Valachovic RW. A survey of dental hygiene program directors: summary findings and conclusions. J Dent Educ. 2009;73(8):1009-32.

11. Aksu MN, Phillips E, Shaefer HL. US dental school deans' attitudes about mid-level providers. J Dent Educ. 2013;77(11):1469-76. Dental schools play an important role in training the new and existing oral health workforce models and therefore the perceptions and attitude of dental deans need to be clearly understood for the success of the models. This first study on the aforementioned phenomena among dental deans clearly illustrate their positions about the models.

12. Lopez N, Blue CM, Self KD. Dental school faculty perceptions of and attitudes toward the new dental therapy model. J Dent Educ. 2012;76(4):383-94. In addition to dental deans, faculty members/educators are vital to the successful training of the oral health workforce models. Thus, the study by Lopez was timely to identify and describe dental school faculty members' perceptions and attitudes of dental therapists.

13. Blue C, Phillips R, Born D, Lopez N. Beginning the socialization to a new workforce model: dental students' preliminary knowledge of and attitudes about the role of the dental therapist. J Dent Educ. 2011;75(11):1465-75. 